\title{
The Role of Complex Electric Permittivity and Magnetic Permeability in Left Handed Materials
}

\author{
Mubarak Dirar Abd Allah', Amna Elzaki Musa ${ }^{2,3}$, Kh. M. Haroun ${ }^{4}$, Ibrahim A.I. Hammad \\ ${ }^{1}$ Sudan University of Science and Technology, Department of Physics, Khartoum, Sudan \\ ${ }^{2}$ Al Jouf University, Department of Physics, Tabarjal, Saudi Arabia \\ ${ }^{3}$ Sinnar University, Department of Physics and Mathematics, Sinnar, Sudan \\ ${ }^{4,5}$ Alzaeim Alazhari University, Department of Physics, Omdurman| Sudan
}

\begin{abstract}
Left handed materials opens new era in modern optics. This motivates trying to build a theoretical model that can help in predicting new materials. The model is based on assuming the existence of medium in electric and magnetic fields. The orientation of medium field with respect to the external ones is described by complex representation. The refractive index is expressed in terms of real and complex electric permittivity and magnetic permeability. It is found that the material becomes left handed if certain restrictions are imposed on the values and orientations of the medium fields with respect to the external ones.
\end{abstract}

Keywords: left handed material, electric permittivity, magnetic, Permeability, refractive index

\section{Introduction}

Refraction is perhaps one of the most basic of electromagnetic phenomena, whereby when a beam of radiation is incident on an interface between two materials at an arbitrary angle, the direction of propagation of the transmitted beam is altered by an amount related to the indices of refraction of the two materials [1]. The material which supports propagation of backward waves ('lefthanded material' or 'backward-wave material') was analyzed theoretically in sixties by Veselago [2] and realized experimentally for the first time by Smith at al. [3] in 2000. Probably the most intriguing electromagnetic phenomenon associated with 'backward-wave' meta-material is 'reversion' of Snell law, i.e. negative refraction. So far, there have been several experimental studies of negative refraction based on pioneering paper published by Shelby et al. [4].

In 1999, Pendry et al. suggested that certain configurations of nonmagnetic conducting elements would have a very strong magnetic response to applied electromagnetic fields. In particular, Pendry et al. predicted that these configurations could have a negative effective permeability over a finite frequency range. As mentioned in Section 1, the magnetic response of naturally occurring materials known to us tails off rapidly with increasing frequency, and in particular is never found to be negative (at least without accompanying large losses). In contrast, if the conducting elements described by Pendry et al. were embedded into some structurally robust host material (e.g., polymer or ceramic), the resulting material could be classified as a material, having a magnetic response not available by other means. Indeed, Pendry et al. suggested a variety of uses for such a, including magnetic shielding. Professor Pendry presented the magnetic structures in 1999 at the Workshop on Electromagnetic Crystal Structures, held in Laguna Beach, California. We became very interested in the experimental demonstration of the magnetic structures, as we felt having the ability to tune the magnetic properties of a surface would be of great significance. To illustrate why a tunable permeability might be of importance, we recall that the reflection coefficient of a wave from a surface has the electric field perpendicular to the plane of incidence (exchange $\varepsilon$ and $\mu$ for electric field parallel to the plane of incidence). The possibility to "match" waves normally incident $\left(\theta_{\mathrm{R}}=0\right)$ from medium 1 to the surface of medium 2 is increased by having control the permeability of the second layer, as there is no reflection for normal incidence. Materials which posses a positive permeability are represented by points in the upper half plane when $e / m$ is the same for both media $[5,6,7]$. For waves incident from other angles, the reflectance can at least be minimized by matching impedance, provided the indices of refraction are also similar in magnitude between the two media. While fabricating the proposed magnetic structures for testing was not a difficult task, unambiguously characterizing the observed scattering behavior as being due distinctly to an inherent permeability represented a challenge. As we initiated a program to construct and characterize test models of the magnetic metamaterial, we realized that the simplest means of demonstrating the negative magnetic permeability was to combine a negative permittivity medium with the magnetic medium and observe the effect of the composite medium on wave propagation $[8,9,10,11,12]$. This work is devoted to find the conditions which are required by the internal fields to make material left handed. This is done in section 2.Sections 3 and 4 are devoted for discussion and conclusion.

\section{Theoretical Model}

The wave number $k$ can be written in terms of the refractive index $n$ in the form.

$$
k=\frac{2 \pi}{\lambda}=\frac{2 \pi f}{\lambda f}=\frac{\omega}{v}=\frac{\omega}{c} \frac{c}{v}=\frac{\omega}{c} n
$$

One can be write the wave number $k$ in a complex form to be 


\section{International Journal of Science and Research (IJSR) \\ ISSN (Online): 2319-7064}

Index Copernicus Value (2016): 79.57 | Impact Factor (2015): 6.391

$$
k=k_{1}+i k_{2}=\frac{\omega}{c}\left(n_{1}+i n_{2}\right)
$$

By equating real and imaginary parts, one gets

$$
k_{1}=\left(\frac{\omega}{c}\right) n_{1} \quad ; \quad k_{2}=\left(\frac{\omega}{c}\right) n_{2}
$$

But the refraction index $\boldsymbol{n}$ can be written in terms of speed of light in free space $\mathbf{c}$ and in the medium $\mathbf{v}$ beside the magnetic permeability $\boldsymbol{\mu}$ and electric permittivity $\boldsymbol{\varepsilon}$ to be:

$$
n=\frac{c}{v}=c \sqrt{\mu \varepsilon}
$$

Splitting $\mathrm{n}$ in to real and imaginary part one gets

Squaring both sides yields

$$
n=n_{1}+i n_{2}
$$

$$
\begin{aligned}
& n^{2}=\left(n_{1}+i n_{2}\right)^{2}=(c \sqrt{\mu \varepsilon})^{2} \\
& n^{2}=n_{1}^{2}+2 n_{1} n_{2} i-n_{2}{ }^{2}=(c \sqrt{\mu \varepsilon})^{2}
\end{aligned}
$$

The magnetic permeability and electric permittivity can also be written in a complex form

$$
\mu=\mu_{1}+i \mu_{2} \quad \varepsilon=\varepsilon_{1}+i \varepsilon_{2}
$$

Inserting Eq. (5) into Eq. (4) yields

$$
\begin{gathered}
n_{1}{ }^{2}-n_{2}{ }^{2}+2 n_{1} n_{2} i=c^{2}\left(\mu_{1}+i \mu_{2}\right)\left(\varepsilon_{1}+i \varepsilon_{2}\right) \\
=c^{2}\left(\mu_{1} \varepsilon_{1}+i \mu_{1} \varepsilon_{2}+i \mu_{2} \varepsilon_{1}-\mu_{2} \varepsilon_{2}\right) \\
=c^{2}\left(\mu_{1} \varepsilon_{1}-\mu_{2} \varepsilon_{2}\right)+i c^{2}\left(\mu_{1} \varepsilon_{2}+\mu_{2} \varepsilon_{1}\right)
\end{gathered}
$$

Equating real and imaginary parts both sides squires

$$
\begin{gathered}
n_{1}{ }^{2}-n_{2}{ }^{2}=c^{2}\left(\mu_{1} \varepsilon_{1}-\mu_{2} \varepsilon_{2}\right) \\
2 n_{1} n_{2}=c^{2}\left(\mu_{1} \varepsilon_{2}+\mu_{2} \varepsilon_{1}\right)
\end{gathered}
$$

$n_{1}$, the real part represents the ordinary refractive index .the imaginary part, $n_{2}$ physical meaning can be understood by recognizing the electric field wave equation and using Eq. (2) to get

$$
\begin{gathered}
E=E_{0} e^{i[(k x-\omega t)}=E_{0} e^{\left.i\left[\left(k_{1}+k_{2}\right) x-\omega t\right)\right]} \\
E=E_{0} e^{i^{2} k_{2} x} \cdot e^{i\left(k_{1} x-\omega t\right)}
\end{gathered}
$$

Thus

$$
E=E_{0} e^{-k_{2} x} e^{i\left(k_{1} x-\omega t\right)}
$$

The electric intensity is given by

Where

$$
I=I_{0} e^{-\alpha x}=|E|^{2}=\left|E_{0}\right|^{2} e^{-2 k_{2} x}
$$

Therefore

$$
I_{0}=E_{0}{ }^{2} \quad I=|E|^{2}
$$

But from Eq. (3)

$$
\alpha=2 k_{2}
$$

$$
k_{2}=\left(\frac{\omega}{c}\right) n_{2}
$$

Hence

$$
\alpha=2\left(\frac{\omega}{c}\right) n_{2}
$$

Thus $n_{2}$ is related to the absorption coefficient $\alpha$ according to the relation.

$$
n_{2}=\frac{c \alpha}{2 \omega}
$$

In view of Eq. (7) and Eq. (10) the refractive index $n_{1}$ is given by.

$$
n_{1}=c^{2}\left(\frac{\mu_{1} \varepsilon_{2}+\mu_{2} \varepsilon_{1}}{2 n_{2}}\right)=2 c w\left(\frac{\mu_{1} \varepsilon_{2}+\mu_{2} \varepsilon_{1}}{\alpha}\right)
$$

This refractive is usually positive but recently some materials, known as left handed materials are shown to have negative refractive index $n_{1}$.In view of Eq. (11), $n_{1}$ is negative when

$$
\begin{gathered}
\left|\mu_{1} \varepsilon_{2}\right|<\left|\mu_{2} \varepsilon_{1}\right| \\
n_{2}=\left(\frac{c \alpha}{2 w}\right)=+\quad \alpha=+, \quad c=+, \quad w=+
\end{gathered}
$$

There are many alternatives that can make the refractive index $n_{1}$ negative, and each alternative requires a certain material structure and arrangement.

In view of Eq. (12). $n_{1}$ is negative, if the following conditions are satisfied, i.e. when

$$
\text { 1. } \varepsilon_{1}=+\varepsilon_{2}=+\mu_{1}=+\mu_{2}=-
$$$$
\text { 2. } \varepsilon_{1}=-\varepsilon_{2}=+\mu_{1}=+\mu_{2}=+
$$

Also $n_{1}$,in Eq. (11) can be negative if

This can be satisfied

$$
\left|\mu_{1} \varepsilon_{2}\right|>\left|\mu_{1} \varepsilon_{2}\right|
$$

$$
\begin{array}{ll}
\text { 1. } \varepsilon_{1}=+\varepsilon_{2}=+\mu_{1}=- & \mu_{2}=+ \\
\text { 2. } \varepsilon_{1}=+\varepsilon_{2}=-\mu_{1}=+ & \mu_{2}=+
\end{array}
$$

The physical meaning of these conditions can be known if the complex representations of $\varepsilon$ and $\mu$ are known. To do this one can write the electric flux density $D$ in terms of the external applied field $E_{e}$ and the complex electric permittivity $\varepsilon$ in the form

$$
\underline{D}=\varepsilon E_{e}=\left(\varepsilon_{1}+i \varepsilon_{2}\right) E_{e}
$$

This can also be viewed also by considering the external field and medium field $E_{m}$ to general the medium flux, i.e.

$$
\underline{D}=\varepsilon E_{e}=\varepsilon_{0}\left(E_{e}+E_{m}\right)
$$

The medium field generally is not parallel to the external one but subtends a certain angle with respect to it.

The component parallel to $E_{e}$ is the real and proportional to it by a factor $c_{1}$.

The component perpendicular to $E_{e}$ is imaginary and proportional to it by a factor $c_{2}$. Thus one can write the medium electric field in the form

$$
E_{m}=c_{1} E_{e}+i c_{2} E_{e}=\left(E_{m_{1}}+i E_{m_{2}}\right)
$$

Inserting Eq. (19) in Eq. (18) yields

$$
D=\varepsilon_{0} E_{e}+\left(\varepsilon_{0} E_{m_{1}}+i \varepsilon_{0} E_{m_{2}}\right)
$$

In view of Eq. (18) one can write $\varepsilon$ in complex form to get

$$
D=\varepsilon_{0}\left[E_{e}+\left(\varepsilon_{1}+i \varepsilon_{2}\right) E_{e}\right]
$$

Taking into account Eq. (20) and Eq. (21), one gets

$$
\begin{gathered}
D=\left[\varepsilon_{0}\left(1+c_{1}\right)+i \varepsilon_{0} c_{2}\right] E_{e} \\
=\left[\varepsilon_{0}\left(1+c_{1}\right)+i \varepsilon_{0} c_{2}\right] E_{e} \\
{\left[\varepsilon_{0}\left(1+c_{1}\right)+i \varepsilon_{0} c_{2}\right] E=\left(\varepsilon_{1}+i \varepsilon_{2}\right) E}
\end{gathered}
$$

Comparing two sides of Eq. (22), it follows that

$$
\varepsilon_{1}=\varepsilon_{0}\left(1+c_{1}\right) \quad \varepsilon_{2}=\varepsilon_{0} c_{2}
$$

The condition in Eq. (14) No (1) is satisfied when

$$
\mu_{2}=-
$$

But what does mean physically?

To understand what happens one have to see Eq. (19), where the reference field is the external electric one $E_{e}$.

Thus the reference magnetic field should be the component $H_{e}$ of the complete electromagnetic wave, which is shown in Fig. (1). 


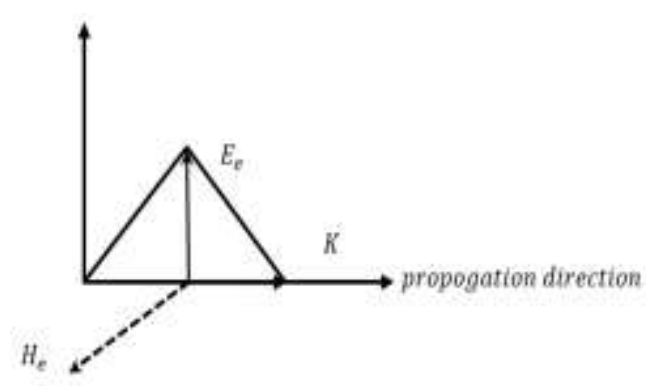

Figure 1: Complete electromagnetic field

Thus the negative of $\mu$ in Eq. (24) means that the internal medium generated magnetic field $H_{m}$ component which is normal to $H_{e}$ subtend an angle $\theta$, such that $\left(\theta=270^{\circ}\right)$ measured into clockwise.

This means that $H_{m}$ lies in the forth quadrant with $\theta$ satisfying

Where

$$
\left(270^{\circ} \leq \theta<360^{\circ}\right)
$$

$$
H_{m}=H_{m_{1}}+i H_{m_{2}}=\mu_{1} H_{m_{1}}+i \mu_{2} H_{m_{2}}
$$

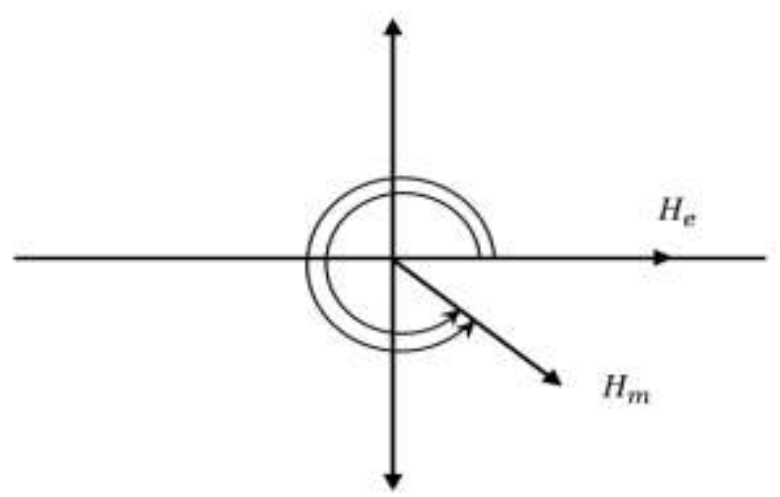

Figure 2: The alignment of the medium magnetic field to make $\mu_{2}$ negative.

The second condition in Eq. (14) requires

This means that

$$
\varepsilon_{1}=\varepsilon_{0}\left(1+c_{1}\right)=-
$$

For $\left|c_{1}\right|>1$

$$
E_{m_{1}}=-\left|c_{1}\right| E_{e}
$$

This requires

$$
\begin{aligned}
& \left|c_{1}\right|=\frac{\left|E_{m_{1}}\right|}{\left|E_{e}\right|}>1 \\
& \quad\left|E_{m_{1}}\right|>\left|E_{e}\right|
\end{aligned}
$$

To find negative refractive index, the component of the medium electric field in the $\boldsymbol{E}$ must align itself in a direction opposite to $\boldsymbol{E}$ and its amplitude $\left|E_{m_{1}}\right|$ need to be more than the amplitude of $\left|E_{e}\right|$ and should be opposite to the direction to $\boldsymbol{E}$.

This means that $\theta_{1}=180^{\circ}$ and $\theta$ satisfies $\left(90^{\circ}<\theta \leq\right.$ $180^{\circ}$, where $\theta$ is measured again anticlockwise.

This can be experimentally done by making atoms or molecules oriented as shown in Fig. (3) and thermally agitated the external field should be make weak such that

$$
\left|E_{m_{1}}\right|>\left|E_{e}\right|
$$

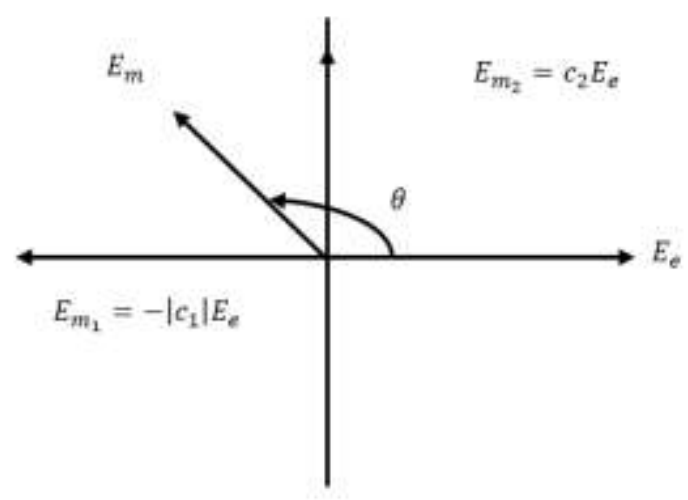

Figure 3: The alignment of $E_{m}$ to make $c_{1}$ negative

Also one can apply a capacitor as shown in Fig. (3)to induce $E_{m}$ by applying alternative electric field.

The second condition which makes $n_{1}$ negative is shown in Eq. (16) No (1) and No (2)

Condition (16) No (1) require that

$$
\mu_{1}=-
$$

But in view of Eq. (25) the medium field is written

$$
H=H_{m_{1}}+i H_{m_{2}}=c_{1} H_{e}+i c_{2} H_{e}
$$

This requires

Thus

$$
\mu_{1}=\mu_{0}\left(1+c_{1}\right)=-
$$

Which means that

$$
\left|c_{1}\right|>1 \quad c_{1}=-\left|c_{1}\right|
$$

$$
\left|c_{1}\right|=\frac{H_{m_{1}}}{H_{e}}>1
$$

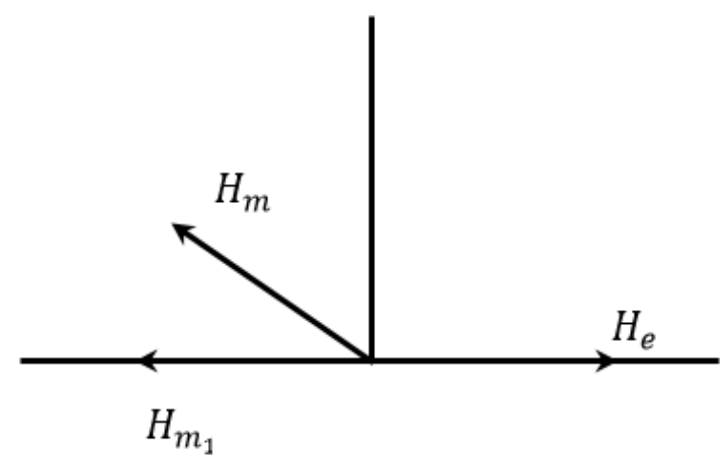

Condition No (1) in Eq. (14) require that

$$
\mu_{2}=-
$$

But in view of Eq. (25) the medium field is written

This requires

$$
H=H_{m_{1}}+i H_{m_{2}}=c_{1} H_{e}+i c_{2} H_{e}
$$

Thus

$$
\mu_{2}=\mu_{0} c_{2}=-
$$

Which means that

$$
c_{2}=-
$$

$c_{2}=-\frac{H_{m_{2}}}{H_{e}}<0$

Condition No (2) in Eq. (16) requires that

$$
\varepsilon_{2}=-
$$

This requires

$$
\varepsilon_{2}=\varepsilon_{0} c_{2}=-
$$


Which means that

$$
c_{2}=-
$$

$c_{2}=-\frac{E_{m_{2}}}{E_{e}}<0$

The refractive index $n_{1}$ given by

$n_{1}=c^{2}\left(\frac{\mu_{1} \varepsilon_{2}+\mu_{2} \varepsilon_{1}}{2 n_{2}}\right)$

For $\mathrm{n}_{2}$ positive $\mathrm{n}_{1}$ can be negative if

1. $\mu_{1}=-\quad \mu_{2}=-\varepsilon_{2}=+\varepsilon_{1}=+$

2. $\mu_{1}=-\quad \varepsilon_{1}=-\quad \mu_{2}=+\quad \varepsilon_{2}=+$

3. $\varepsilon_{2}=-\mu_{2}=-\quad \varepsilon_{1}=+\quad \mu_{1}=+$

$$
\text { 4. } \varepsilon_{2}=-\quad \varepsilon_{1}=-\quad \mu_{1}=+\quad \mu_{2}=+
$$

Condition No (1) in Eq. (39) requires that

$$
\mu_{1}=-
$$

$\begin{aligned} \mu_{1} & =-\end{aligned}$

$$
H=H_{m_{1}}+i H_{m_{2}}=c_{1} H_{e}+i c_{2} H_{e}
$$

This requires

Thus

$$
\mu_{1}=\mu_{0}\left(1+c_{1}\right)=-
$$

Which means that

$$
\begin{array}{r}
\left|c_{1}\right|>1 \\
c_{1}=-\left|c_{1}\right|
\end{array}
$$

$\left|c_{1}\right|=\frac{H_{m_{1}}}{H_{e}}>1$

$$
\mu_{2}=-
$$

But in view of Eq. (25) the medium field is written

$$
H=H_{m_{1}}+i H_{m_{2}}=c_{1} H_{e}+i c_{2} H_{e}
$$

This requires

Thus

$$
\mu_{2}=\mu_{0} c_{2}=-
$$

$$
c_{2}=-
$$

Which means that

$$
c_{2}=-\frac{H_{m_{2}}}{H_{e}}<0
$$

Condition No (2) in Eq. (39) requires that

$$
\mu_{1}=-
$$

But in view of Eq. (25) the medium field is written

$$
H=H_{m_{1}}+i H_{m_{2}}=c_{1} H_{e}+i c_{2} H_{e}
$$

This requires

Thus

$$
\mu_{1}=\mu_{0}\left(1+c_{1}\right)=-
$$

$$
\begin{aligned}
& \left|c_{1}\right|>1 \\
& c_{1}=-\left|c_{1}\right|
\end{aligned}
$$

Which requires that

$$
\begin{array}{r}
\left|c_{1}\right|=\frac{H_{m_{1}}}{H_{e}}>1 \\
\varepsilon_{1}=-
\end{array}
$$

This requires

Thus

$$
\varepsilon_{1}=\varepsilon_{0}\left(1+c_{1}\right)=-
$$

Which requires that

$$
c_{1}=-\left|c_{1}\right|>1
$$

$\left|c_{1}\right|=\frac{\left|E_{m_{1}}\right|}{\left|E_{e}\right|}>1$

Condition No (3) in Eq. (39) requires that

This requires

$$
\varepsilon_{2}=-
$$

Thus

$$
\varepsilon_{2}=\varepsilon_{0} c_{2}=-
$$

Which means that

$c_{2}=\frac{E_{m_{2}}}{E_{e}}<0$

$$
\mu_{2}=-
$$

But in view of Eq. (25) the medium field is written

$$
H=H_{m_{1}}+i H_{m_{2}}=c_{1} H_{e}+i c_{2} H_{e}
$$

This requires

Thus

$$
\begin{gathered}
\mu_{2}=\mu_{0} c_{2}=- \\
c_{2}=-
\end{gathered}
$$

Which means that

$$
c_{2}=\frac{H_{m_{2}}}{H_{e}}<0
$$

Condition No (4) in Eq. (39) requires that

This requires

$$
\varepsilon_{2}=-
$$

Thus

$$
\varepsilon_{2}=\varepsilon_{0} c_{2}=-
$$

Which means that

$$
c_{2}=-
$$

Which means that

$$
\begin{gathered}
c_{2}=\frac{E_{m_{2}}}{E_{e}}<0 \\
\varepsilon_{1}=-
\end{gathered}
$$

This requires

$$
\varepsilon_{1}=\varepsilon_{0}\left(1+c_{1}\right)=-
$$

Thus

$$
\left|c_{1}\right|>1
$$

Which requires that

$$
c_{1}=-\left|c_{1}\right|>1
$$

\section{Discussion}

The theoretical conditions to have left handed materials ,which requires $n_{1}$ to be negative is exhibited in section (2)the refractive index is given according to Eq. (11) in terms of real and imaginary electric permittivity and magnetic permeability. To understand the physical meaning of these quantities, it is better to refer to Eq. (7) and Eq (10), where the absorption coefficient $\alpha$ is given by

$$
\alpha=\frac{c w}{n_{1}}\left(\frac{\mu_{1} \varepsilon_{2}+\mu_{2} \varepsilon_{1}}{\alpha}\right)
$$

In the case that the electric generated medium field is parallel to the external one

and

$$
\varepsilon_{2}=0
$$

$$
\alpha=\frac{\mathrm{cw}}{\mathrm{n}_{1}} \varepsilon_{1} \mu_{2}
$$

Thus the imaginary magnetic permeability $\boldsymbol{\mu}_{2}$ is directly responsible for absorption of light. This is since atoms absorb photon then reemit part of it in the original photon direction while the other part having magnetic field perpendicular to the incident one is absorbed by the medium. However, when the generated medium magnetic component of light is in the same direction as the incidence one $\mu_{2}=0$

Thus

$$
\alpha=\frac{\mathrm{cw}}{\mathrm{n}_{1}} \mu_{1} \varepsilon_{2}
$$

Which means that the imaginary permittivity $\varepsilon_{2}$ is responsible for absorption. This is again due to the fact that the absorbed incident photon is re emitted by the atom such 


\section{International Journal of Science and Research (IJSR) \\ ISSN (Online): 2319-7064}

Index Copernicus Value (2016): 79.57 | Impact Factor (2015): 6.391

that part of it is absorbed by the medium. The absorption process is similar to that discussed for the magnetic field. The material become left handed according to No (1) in Eq. (14) if imaginary magnetic permittivity is negative .This physically means that the medium magnetic component which is perpendicular to the applied external magnetic field is anti parallel to the external electric field. In view of No (2) in Eq. (14) the material become left handed if the medium electric field is anti parallel to the external one .This make $\varepsilon_{1}$ negative.

According to Eq. (15) and Eq. (16) the material become left handed if the induced medium electric field component which is parallel to the incident one and the induced absorbed magnetic field are very large [see No (1) in Eq. (16)], such that the medium magnetic component $\mu_{1}$ is anti parallel to the external one. Also the material can be made left handed if the medium generated electric field is anti parallel to the direction of the external magnetic field.

In view of Eq. (11) and Eq. (39) there are additional conditions to have left handed materials .According to No (1) in Eq. (39) the left handed material requires both $\mu_{1}$ and $\mu_{2}$ to be negative. This means that the components of the medium magnetic fields need to be anti parallel to the external magnetic and external electric field respectively.

The material becomes left handed according to No (2) in Eq. (39) if imaginary magnetic permittivity and electric permeability are negative.

This physically means that the medium magnetic component which is perpendicular to the applied external magnetic field and external electric field are anti parallel to the external electric field and external magnetic field respectively.

According to No (3) in Eq. (39) the left handed material requires both $\varepsilon_{2}$ and $\mu_{2}$ to be negative. This means that the components of the medium magnetic fields need to be anti parallel to the external magnetic and external electric field respectively.

The material becomes left handed according to No (4) in Eq. (39) if imaginary magnetic permittivity and electric permeability are negative. This physically means that the medium magnetic component which is perpendicular to the applied external magnetic field and external electric field are anti parallel to the external electric field and external magnetic field respectively.

\section{Conclusion}

The material becomes left handed according to the orientation of medium electric and magnetic field with respect to the external electric and magnetic components of the electromagnetic waves.

\section{References}

[1] Shelby, R. A., D. R. Smith, S. Schultz, Experimental verification of a negative index of refraction, Science, Vol. 292, No. 6, 77-79, April (2001). http://web.pdx.edu/ larosaa/Applied_Optics_464564/Pr
ojects_Optics/Plasmons/2001_Experimental_Verificatio n_of\%20Negative_index_of_refraction.pdf

[2] V. G. Veselago, The Electrodynamics of Substances with Simultaneously Negative Values of $\varepsilon$ and $\mu$ ,USPEKHI, vol. 10, No 4, pp. 509-514, JanuaryFebruary (1968). http://iopscience.iop.org/article/10.1070/PU1968v010n0 4ABEH003699/meta

[3] 3] D.R. Smith et. al., A Composite Media with Simultaneously Negative Permeability and Permittivity, App. Physics Letters, Vol 84, No 18, pp 4184-4187, May http://muri.lci.kent.edu/References/NIM_Papers/Nanop article_Theory_Experiment/2000_SRR_Properties.pdf

[4] S. Hrabar, Z. Sipus and J. Bartolic, Experimental verification of negative index of refraction by lateral beam displacement, Department of Radiocommunications and Microwave Engineering,University of Zagreb Unska 17, Zagreb, HR $10 \quad 000, \quad$ Croatia. https://pdfs.semanticscholar.org/ef96/31b32170c5dfc96 301592068b0dcb6e9b29e.pdf

[5] Veselago, V. G., The electrodynamics of substances with simultaneously negative values of $\varepsilon$ and $\mu$, Sov. Phys. Uspe., Vol. 10, No. 4, 509-514, July (1968). http://elibrary.lt/resursai/Uzsienio\%20leidiniai/MFTI/V eselago/Trudy/Thaormina.pdf

[6] Pendry, J. B., Negative refraction makes a perfect lens, Phys. Rev. Lett., Vol. 85, No. 18, 3966-3969, October (2000).

http://citeseerx.ist.psu.edu/viewdoc/download?doi=10.1. $1.730 .7922 \&$ rep $=$ rep $1 \&$ type $=$ pdf

[7] Zhang, X. and Z. Liu, Super lens to overcome the diffraction limit, Nature Mater. Vol. 7, 435-441, June (2008). https://www.nature.com/articles/nmat2141

[8] Aydin, K., I. Bulu and E. Ozbay, Sub wavelength resolution with a negative index metamaterial super lens, Appl. Phys. Lett., Vol. 90, No. 254102, June (2007).http://yoksis.bilkent.edu.tr/pdf/files/10.10631.2750393.pdf

[9] Fang, N. and X. Zhang, Imaging properties of a metamaterial Super lens, Appl. Phys. Lett., Vol. 82, No. 2 , 161-163, January (2003).http://xlab.me.berkeley.edu/MURI/publications/ publications_22.pdf

[10] Iyer, A. K. and G. V. Eleftheriades, Free-space imaging beyond the diffraction limit using a Veselago-Pendry transmission-line metamaterial super lens, IEEE Trans. Antennas Propagate., Vol. 57, No. 6, 1720-1727, June (2009).https://arxiv.org/ftp/arxiv/papers/0803/0803.201 7.pdf

[11] Smith, D. R., J. B. Pendry, and M. C. K. Wiltshire, Metamaterials and negative refractive index, Science, Vol. 305, 788-792, Aug. (2004) http://www.cmth.ph.ic.ac.uk/photonics/Newphotonics/p df/ScienceReviewReprint.pdf

[12]Ziolkowski, R. W. and E. Heyman, Wave propagation in media having negative permittivity and permeability, Phys. Rev. E, Vol. 64, No. 056625, 1-15, October (2001).

https://pdfs.semanticscholar.org/a2dd/dd19d4b28ab235 4b1ef3653af04ec1c55f16.pdf 